\title{
Sonic Hedgehog Guides Post-Crossing Commissural Axons Both Directly and Indirectly by Regulating Wnt Activity
}

\author{
Elena Domanitskaya, ${ }^{1 \star}$ Andrin Wacker, ${ }^{1 \star}$ Olivier Mauti, ${ }^{1}$ Thomas Baeriswyl, ${ }^{1}$ Pilar Esteve, ${ }^{2}$ Paola Bovolenta, ${ }^{2}$ \\ and Esther T. Stoeckli ${ }^{1}$ \\ ${ }^{1}$ Institute of Molecular Life Sciences, University of Zurich, 8057 Zurich, Switzerland, and ${ }^{2}$ Instituto Cajal, CSIC and CIBER de Enfermedades Raras, 28002 \\ Madrid, Spain
}

After midline crossing, axons of dorsolateral commissural neurons turn rostrally into the longitudinal axis of the spinal cord. In mouse, the graded distribution of Wnt 4 attracts post-crossing axons rostrally. In contrast, in the chicken embryo, the graded distribution of Sonic hedgehog $(\mathrm{Shh})$ guides post-crossing axons by a repulsive mechanism mediated by hedgehog-interacting protein. Based on these observations, we tested for a possible cooperation between the two types of morphogens. Indeed, we found that Wnts also act as axon guidance cues in the chicken spinal cord. However, in contrast to the mouse, Wnt transcription did not differ along the anteroposterior axis of the spinal cord. Rather, Wnt function was regulated by a gradient of the Wnt antagonist Sfrp1 (Secreted frizzled-related protein 1) that in turn was shaped by the Shh gradient. Thus, Shh affects post-crossing axon guidance both directly and indirectly by regulating Wnt function.

\section{Introduction}

Commissural neurons in the dorsolateral spinal cord (dI1 neurons) extend their axons ventromedially toward the floor plate, the ventral midline of the spinal cord (Avraham et al., 2009). After midline crossing, axons turn rostrally along the contralateral floor-plate border. The molecular mechanisms that guide commissural axons toward the floor plate have been well characterized. Guidance cues for post-crossing commissural axons have been identified only more recently (Lyuksyutova et al., 2003; Bourikas et al., 2005; Niederkofler et al., 2010). In the mouse, an attractive effect of Wnt 4 on post-crossing commissural axons was demonstrated in explant cultures (Lyuksyutova et al., 2003). In the chicken embryo, we identified a different morphogen, Sonic hedgehog (Shh), as an axon guidance cue for post-crossing commissural axons both in vivo and in vitro (Bourikas et al., 2005).

In agreement with its graded expression in the mouse floor plate, Wnt4 was found to act as an attractant. High Wnt4 mRNA expression was found in the floor plate at rostral and low expression at more caudal levels (Lyuksyutova et al., 2003). In contrast, Shh was found to act as a repellent for post-crossing axons, consistent with its expression pattern characterized by high levels of Shh in the caudal floor plate and low levels more rostrally (Bourikas et al., 2005). Thus, Shh has a dual role in commissural axon guidance. First, it acts as a chemoattractant in parallel to Netrin-1

Received March 23, 2010; revised June 30, 2010; accepted July 3, 2010.

This work was supported by grants of the Swiss National Science Foundation and the NCCR Brain Plasticity and Repair. We thank S. Arber, L. Burrus, A. Klar, C. Krull, and M. Tessier-Lavigne for reagents and members of the laboratory for critically reading the manuscript.

*E.D. and A.W. contributed equally to this work.

Correspondence should be addressed to Esther T. Stoeckli, Institute of Molecular Life Sciences, University of Zurich, Winterthurerstrasse 190, 8057 Zurich, Switzerland. E-mail: Esther.Stoeckli@imls.uzh.ch.

E. Domanitskaya's present address: Department of Molecular Biology, Princeton University, Washington Road, Princeton, NJ 08544.

DOI:10.1523/JNEUROSCI.1488-10.2010

Copyright $\odot 2010$ the authors $\quad 0270-6474 / 10 / 3011167-10 \$ 15.00 / 0$
(Charron et al., 2003), then, only a few hours later, Shh switches from attractant to repellent and pushes post-crossing axons rostrally (Bourikas et al., 2005). This change in activity is possibly due to a switch in receptors. While precommissural axons are growing toward the floor plate, they are attracted by Shhmediated Smo (Smoothened) (Charron et al., 2003; Yam et al., 2009) and Boc [Brother of Cdo (Cell-adhesion molecule-related, downregulated by oncogenes)] (Okada et al., 2006). After reaching the midline, they no longer express Ptc (Patched) and Smo but use hedgehog-interacting protein (Hhip) as the receptor that mediates the repulsive response to Shh (Bourikas et al., 2005).

These findings raised the question whether Wnts and Shh would cooperate in post-crossing commissural axon guidance (Stoeckli, 2006). To address this issue, we explored the expression pattern of Wnts in the embryonic chicken spinal cord. Based on their temporal and spatial expression pattern, Wnt5a and Wnt7a were good candidate guidance cues for post-crossing axons. Functional analysis by in ovo RNA interference (RNAi) did indeed demonstrate their involvement in axon guidance along the longitudinal axis of the lumbosacral spinal cord. Interestingly, we found no evidence for a Wnt expression gradient in the chicken spinal cord. Rather Wnt activity gradually decreased toward the caudal spinal cord by a gradient of Secreted frizzled-related protein (Sfrp)1, a known Wnt antagonist. Because expression of Sfrps can be regulated by Shh, Shh determines post-crossing commissural axon guidance both directly (Bourikas et al., 2005) and indirectly through regulating the effects of Wnts (this study).

\section{Materials and Methods}

Preparation of in situ probes and double-stranded RNA. Probes for in situ hybridization and double-stranded RNA (dsRNA) were produced from the following chicken expressed sequence tags: ChEST17914 [Wnt4, base pair 268-1177 of the open reading frame (ORF) and $121 \mathrm{bp}$ of $\left.3^{\prime}-\mathrm{UTR}\right]$, ChEST2k9 (Wnt5a, base pair 6-823 of the ORF), ChEST809e5 (Wnt5b), ChEST543m22 (Wnt7a, base pair 421-1050), ChEST661e23 (Wnt7b), 
ChEST421c6 (Wnt8a), ChEST530d5 (Wnt9b), ChEST41h24 (Wnt11, 903 base pair of 3' UTR), ChEST763j19 (Sfrp2, base pair 195867), and ChEST108h20 (Sfrp3, base pair 4951065 of the ORF and 465 base pair of the 3'-UTR) (Geneservice). Plasmids containing these fragments were linearized with NotI and EcoRI (New England Biolabs). The Sfrp1 plasmid (750 bp of the $3^{\prime}$-UTR) was linearized with BamHI and EcoRV (New England Biolabs). Digoxigenin-labeled probes were prepared and used for in situ hybridization as described previously (Mauti et al., 2006). dsRNA was generated by in vitro transcription as described previously (Pekarik et al., 2003). All sequences were carefully analyzed to avoid overlapping stretches that could lead to downregulation of nontargeted family members.

Efficiency and specificity of target gene downregulation was verified in cryosections of the lumbosacral spinal cord of embryos at stages (HH) 25-26, as described by Hamburger and Hamilton (1951). In the absence of specific antibodies for Wnts and Sfrps, we used in situ hybridization to measure downregulation of the target mRNAs (Mauti et al., 2007). For each condition, at least five sections from three to four embryos were quantified using ImageJ software (supplemental Fig. S1, available at www.jneurosci.org as supplemental material).

In ovo RNAi. Fertilized eggs (Hisex, Hendrix Genetics) were obtained from a local hatchery. The eggs were incubated at $38.5^{\circ} \mathrm{C}$ until the embryos reached the desired developmental stage (Hamburger and Hamilton, 1951). For functional analyses, plasmids or dsRNA for in ovo RNAi were injected and electroporated as detailed previously (Pekarik et al., 2003; Bourikas et al., 2005). In brief, a solution containing $300 \mathrm{ng} / \mu \mathrm{l}$ dsRNA derived from the gene of interest and a plasmid encoding enhanced green fluorescent protein (EGFP) under the control of the $\beta$-actin promoter $(50 \mathrm{ng} / \mu \mathrm{l})$ were injected into the central canal of the spinal cord of HH18/19 embryos. Transfection of the floor plate or one-half of the spinal cord was achieved by electroporation with five pulses at $26 \mathrm{~V}$ with a $1 \mathrm{~s}$ interpulse interval (BTX Electro Square Porator ECM830) (supplemental Fig. S2, available at www.jneurosci.org as supplemental material) (Bourikas et al., 2005). Our analysis of post-crossing commissural axon pathfinding was restricted to the lumbosacral level of the spinal cord. Only dye-injection sites that were in the EGFP-positive areas of the spinal cord were considered for further analysis (supplemental Fig. S2, available at www. jneurosci.org as supplemental material). Embryos injected with the EGFP plasmid alone were used as controls and compared with untreated embryos.

Analysis of neural tube patterning. For the analysis of neural tube patterning, we used 25$\mu \mathrm{m}$-thick cryosections of the lumbosacral spinal cord of nontreated and experimental embryos. Immunostaining was done as described previously (Perrin et al., 2001). The induction of ventral and dorsal cell types was assessed by Nkx2.2 (74.5A5), Pax7, Islet-1 (40.2D6), HNF3 $\beta$ (4C7), and Shh (5E1) staining. Monoclonal antibodies devel-
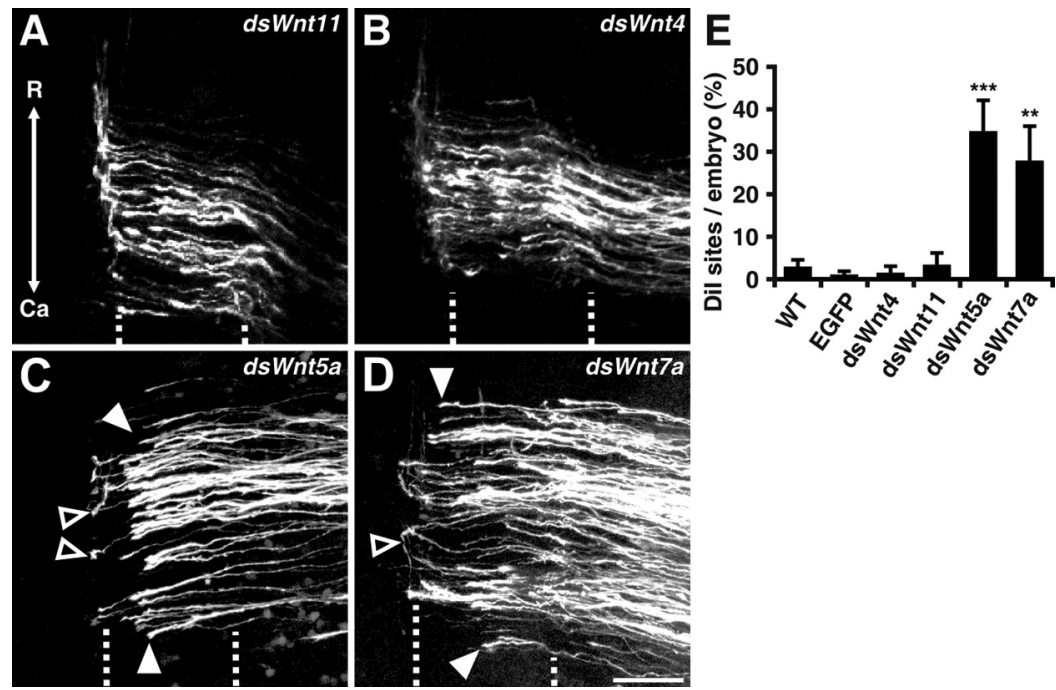

Figure 1. Wnt5a and Wnt7 guide post-crossing commissural axons rostrally. $\boldsymbol{A}-\boldsymbol{D}$, We used in ovo RNAi to downregulate Wnts in the floor-plate area during commissural axon pathfinding. Neither Wnt11 dsRNA (dsWnt11; $\boldsymbol{A})$ nor Wnt4 dsRNA (dsWnt4; $\boldsymbol{B})$ injection and electroporation had an effect on post-crossing commissural axon guidance. In contrast, downregulation of Wnt5a $(d s W n t 5 a ; C)$ resulted in the majority of axons stalling in the floor plate. Those axons that reached the contralateral floor-plate border randomly turned rostrally (filled arrowheads) or caudally (open arrowheads). Similarly, axons failed to turn rostrally in the absence of Wnt7a $(d s W n t 7 a ; \boldsymbol{D})$. In general, more axons reached the contralateral floor-plate border in the absence of Wnt7a compared with Wnt5a. Injection sites with $>50 \%$ of the axons stalling in the floor plate or at the floor-plate exit site, or with axons turning caudally, were considered to exhibit a severe phenotype. The percentage of injection sites with severe phenotypes was calculated per embryo (supplemental Table S1, available at www.jneurosci.org as supplemental material). Scale bar, $40 \mu \mathrm{m}$. E, At least 11 embryos for each experimental condition were used for quantification. In the absence of Wnt5a, a strong phenotype was observed, on average, at $34.9 \pm 7.2 \%$ of the injection sites per embryo ( $n=11$ embryos). In the absence of Wnt7a, a strong phenotype was found, on average, at $27.9 \pm 8.1 \%$ of the injection sites $(n=16$ embryos). The values found after downregulation of Wnt4 (1.5 $\pm 1.5 \%, n=11$ embryos) or Wnt11 (3.4 $\pm 2.7 \%, n=11$ embryos) were not different from controls (wild type; WT). Untreated control embryos ( $3.0 \pm 1.6 \%, n=11$ embryos) did not differ from EGFP-expressing embryos $(1.1 \pm 0.8 \%, n=18$ embryos). Values are given \pm SEM. A two-tailed Student's $t$ test was used for statistical analysis. ${ }^{* * *} p<0.001$ for Wnt5a versus all control groups; ${ }^{* *} p<0.01$ for Wnt7a versus EGFP controls. The $p$ value was $<0.05$ for Wnt7a versus nontreated controls and versus embryos lacking Wnt4 or Wnt11, respectively (supplemental Table S1, available at www.jneurosci.org as supplemental material). R, Rostral; (a, caudal.
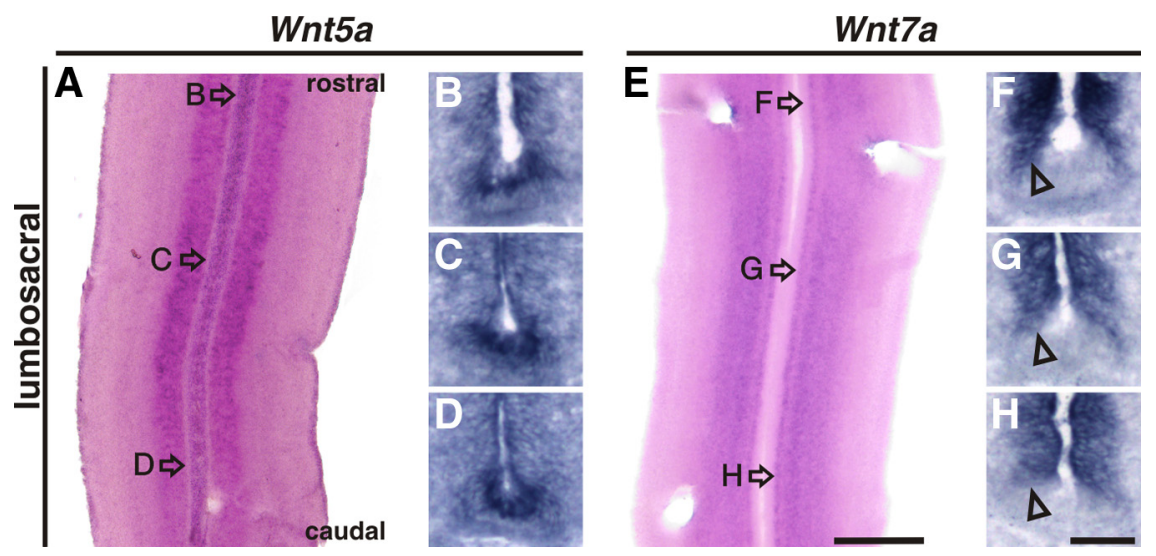

Figure 2. Neither Wnt5a nor Wnt7a is expressed in a gradient along the anteroposterior axis of the chicken lumbosacral spinal cord. $\boldsymbol{A}-\boldsymbol{H}$, Open-book preparations $(\boldsymbol{A}, \boldsymbol{E})$ and transverse sections $(\boldsymbol{B}-\boldsymbol{D}, \boldsymbol{F}-\boldsymbol{H})$ of $\mathrm{HH} 26$ spinal cords were used for in situ hybridization analyses of $W n t 5 a(\boldsymbol{A}-\boldsymbol{D})$ and $W n t 7 a(\boldsymbol{E}-\boldsymbol{H})$ expression. No gradient was detectable for $W n t 5 a \mathrm{mRNA}$ in the floor plate of the lumbosacral region (supplemental Fig. S5, available at www.jneurosci.org as supplemental material), although slightly higher levels were found at the thoracic level (data not shown). Expression of Wnt5a in motoneurons is restricted to lumbosacral levels of the spinal cord. No gradient was observed for Wnt7a in the area adjacent to the floor plate $(\boldsymbol{F}-\boldsymbol{H}$, open arrowhead). Arrows in $\boldsymbol{A}$ and $\boldsymbol{E}$ indicate levels of transverse sections shown in $\boldsymbol{B}-\boldsymbol{D}$ and $\boldsymbol{F}-\boldsymbol{H}$. Scale bars: (in $\boldsymbol{H}) \boldsymbol{B}-\boldsymbol{D}, \boldsymbol{F}-\boldsymbol{H}, 50 \mu \mathrm{m}$; (in $\boldsymbol{E}) \boldsymbol{A}, \boldsymbol{E}, 500 \mu \mathrm{m}$.

oped by T. Jessell, S.Brenner-Morton, and A. Kawakami were obtained from the Developmental Studies Hybridoma Bank established under the auspices of the National Institute of Child Health and Human Development and maintained by The University of Iowa. Nkx2.2 and Pax7 anti- 

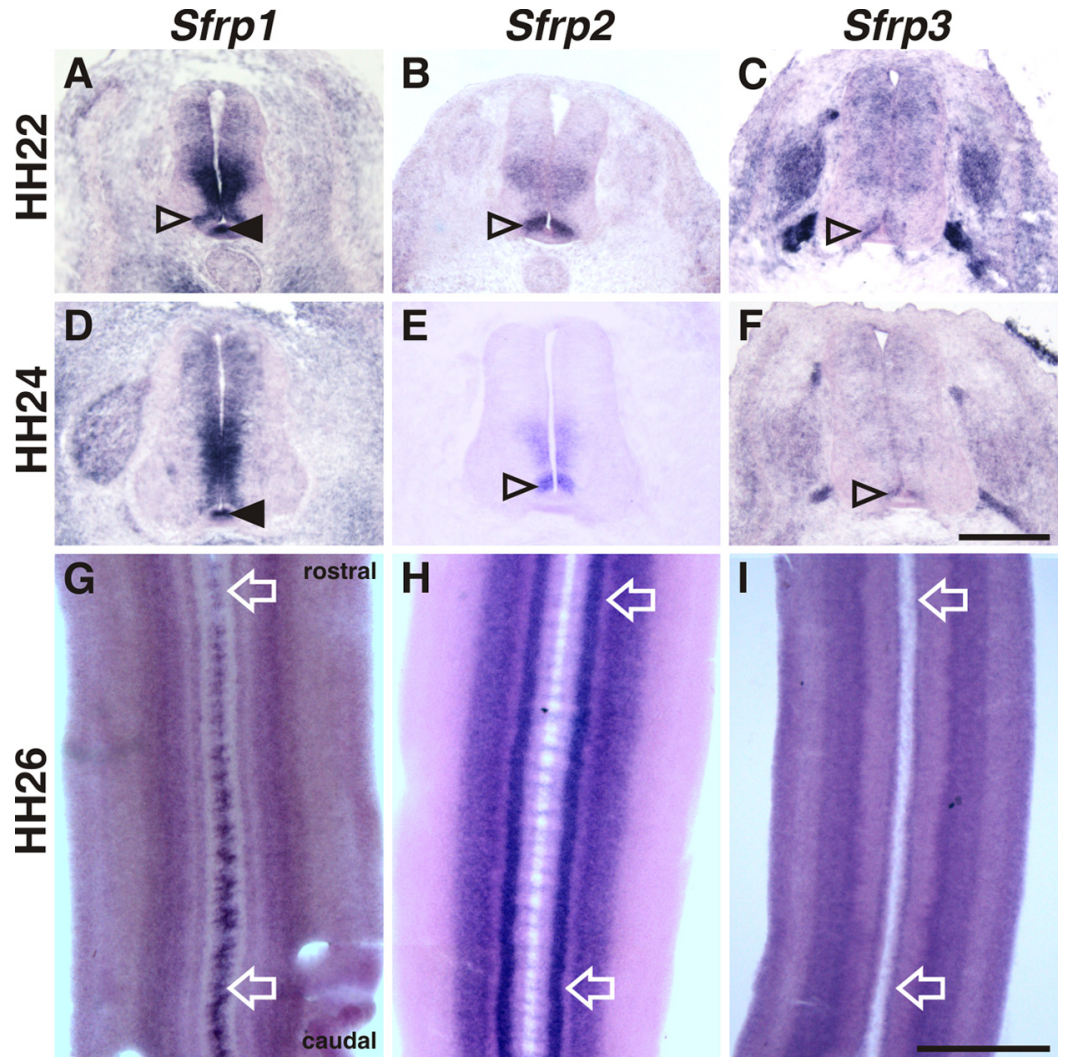

Figure 3. Expression patterns of Sfrps in the embryonic chicken spinal cord. A, D, G, Sfrp 1 is expressed in the floor plate (filled arrowhead), in the ventricular zone, and in an area dorsolateral to the floor plate (open arrowhead) at $\mathrm{HH} 22$ (A), HH24 (D), and HH26 (G). $\boldsymbol{B}, \boldsymbol{E}, \boldsymbol{H}$, Sfrp2 expression was found in the ventral ventricular zone with an area of stronger expression dorsal to the floor plate (open arrowhead). $\boldsymbol{C}, \boldsymbol{F}, \boldsymbol{I}$, Sfrp 3 is expressed in an area adjacent to the floor plate ( $\boldsymbol{C}$ and $\boldsymbol{F}$, open arrowheads; $\boldsymbol{I}$ ) similar to Wnt7a. In contrast to Wnts, a strong gradient of Sfrp 1 was found in the floor plate along the anteroposterior axis with high levels in the caudal floor plate (G) (supplemental Fig. S5, available at www.jneurosci.org as supplemental material). Sfrp2 was expressed in a shallow gradient, in contrast to Sfrp3 that was not expressed in a gradient along the anteroposterior axis. Compare expression indicated by arrows in $\mathbf{G}-\boldsymbol{I}$ (supplemental Fig. 55 , available at www.jneurosci.org as supplemental material). Rostral is to the top in $\mathbf{G}-\boldsymbol{I}$. Scale bars: (in $\boldsymbol{F}) \boldsymbol{A}-\boldsymbol{F}, 200 \mu \mathrm{m}$; (in $\boldsymbol{I}) \mathbf{G}-\boldsymbol{I}, 500 \mu \mathrm{m}$.

bodies were mixed for staining of transverse spinal-cord sections after downregulation or overexpression of Sfrps and Wnts. Fluorescent secondary antibodies [goat anti-mouse IgG-Cy3 (Jackson ImmunoResearch) and goat anti-rabbit Alexa350 (Invitrogen)] were used at a dilution of 1:250.

Analysis of post-crossing commissural axon pathfinding. The analysis of commissural axon trajectories in the lumbosacral spinal cord was performed as described previously (Stoeckli and Landmesser, 1995; Perrin and Stoeckli, 2000; Bourikas et al., 2005). Chicken embryos were killed between HH25 and HH26 (Hamburger and Hamilton, 1951), the spinal cord was removed, opened at the roof plate (open-book preparation), and fixed for $30 \mathrm{~min}$ in $4 \%$ paraformaldehyde (PFA) in PBS at room temperature. The lipophilic dye Fast-DiI ( $5 \mathrm{mg} / \mathrm{ml}$ in methanol; Invitrogen) was applied to the cell bodies of dorsolateral commissural neurons (dI1 neurons). To allow for diffusion of the dye, the open-book preparations were kept in $\mathrm{PBS}$ at $4^{\circ} \mathrm{C}$ for $2-3 \mathrm{~d}$. The spinal cords were mounted in PBS between two coverslips sealed with high-vacuum grease (Dow Corning). The phenotypes were classified as normal (axons turn rostrally along the contralateral floor-plate border and no more than $20 \%$ of the axons stall within the floor plate), weak (stalling of $20-50 \%$ of the axons before reaching the contralateral floor-plate border), and strong (commissural axons found to turn caudally or $>50 \%$ of the axons stalling in the floor plate or at the floor-plate exit site). Caudal turns were never observed when only dorsolateral commissural axons were analyzed as done here and in our previous studies (Bourikas et al., 2005). On average, seven injection sites per spinal cord were analyzed. Only DiI injection sites where the dye was exclusively applied to the area of the dorsolateral border cells were analyzed. Injection sites where the dye spread too ventrally were not considered, as this may have labeled more ventrally located populations of commissural neurons with divergent axonal trajectories (Avraham et al., 2009). Only embryos with more than three injection sites that fulfilled these criteria were included in the quantitative analysis.

Ectopic expression of Wnts and Sfrps. For gain-of-function experiments, the open reading frames of chicken Wnt5a and Wnt7a were cloned in the pMES vector (kindly provided by C. Krull, University of Michigan, Ann Arbor, MI). Total RNA was purified from spinal cords of HH25/26 chicken embryos using TRIzol (Invitrogen) according to the manufacturer's protocol and used as template for cDNA synthesis with the SuperScript Choice System (Invitrogen) and the T7-(T)24 primer 5'-GGCCAGTGAATTGTAATACGACTCACTATAGGGAGGCG$\mathrm{G}(\mathrm{dT}) 24-3^{\prime}$. Specific primers for $W n t 5 a$ were 5' -CTAGTCTAGAATGGAGAAATCCACTGCAGTATTAA-3' (forward) and 5'-CGGAATTCCTATTTGCACACAAACTGGTCC-3' (reverse). For Wnt7a cloning, the forward primer was $5^{\prime}$-CTAGTCTAGAATGAACAGGAAAACAAGGC- $3^{\prime}$ and the reverse primer was 5'-CGGAATTCTCACTTACAGGTATATACTTCTGTT-3'. XbaI and EcoRI restriction sites were introduced to the forward and reverse primers for cloning the PCR fragments into the pMES vector. The pMES plasmid contains an internal ribosome entry site (IRES) sequence followed by EGFP, thus allowing for direct detection of transfected cells. The pCIG-Sfrp1-myc/his-IRES-EGFP plasmid was generated from the pCDNA3.1-Sfrp1-myc/his expression vector (Esteve et al., 2003). pCIGSfrp2-myc/his and pCIG-Sfrp3-myc/his plasmids were kindly provided by Laura Burrus (San Francisco State University, San Francisco, CA). In the pCIG plasmid, the IRES sequence is followed by EGFP containing a nuclear localization sequence. The pMES-Shh was described previously (Bourikas et al., 2005). For overexpression, we injected $500 \mathrm{ng} / \mu \mathrm{l}$ of the plasmid. When the plasmid did not contain an IRES sequence followed by EGFP, we coinjected $50 \mathrm{ng} / \mu \mathrm{l}$ of a plasmid encoding EGFP under the control of the $\beta$-actin promoter to visualize transfected cells. To reverse the functional gradient of endogenous Wnt5a and Wnt7a, the respective plasmids were injected into the central canal of the embryonic spinal cord and electrodes were positioned at caudal levels of the lumbosacral spinal cord. For the ectopic expression of Sfrps, the electrodes were positioned at thoracic levels.

In vitro assays. For cultures of post-crossing commissural axons, spinal cords were dissected at $\mathrm{HH} 25$ as described previously (Bourikas et al. 2005). Explants were cultured in collagen gels alone or with COS7 cells that were transfected with Lipofectamine 2000 (Invitrogen) either with an empty vector (pcDNA3.1; Invitrogen) or with pcDNA3.1 containing the cDNA of Wnt $5 a$ or $W n t 7 a$ (for details about cloning, see above). After $20-24 \mathrm{~h}$, cells were fixed in 4\% PFA for $1 \mathrm{~h}$. Axons were visualized by staining with $2 \mathrm{U} / \mathrm{ml}$ Oregon Green phalloidin for $20 \mathrm{~min}$. The explants were analyzed with ImageJ as described by Wolf and colleagues (2008). Axon growth of experimental explants was normalized to control explants (without cells) for each experiment. Values from three to five experiments were pooled and $p$ values calculated with Student's $t$ test (two-tailed distributions). Wnt activity was blocked by adding recombinant human Sfrp1 (R\&D Systems) to a final concentration of $1 \mu \mathrm{g} / \mathrm{ml}$.

To demonstrate that axons extending from these explants are indeed post-crossing commissural axons, we used a construct for the expression of MARCKS-EGFP (kindly provided by S. Arber, University of Basel, 
Basel, Switzerland) under the control of the Math1-promoter that is expressed specifically in dorsal commissural axons (data not shown).

For cultures of pre-crossing commissural axons, chicken spinal cords were dissected at $\mathrm{HH} 22 / 23$ and explants of dorsal spinal cord (without floor plate) were cultured for 20-24 h alone or with either mock-transfected or Wntexpressing COS7 cells. As a positive control, HEK293T cells stably expressing Netrin-1 (kindly provided by Dr. M. Tessier-Lavigne, Genentech, South San Francisco, CA) were used.

To demonstrate the graded activity of Wnts along the anteroposterior axis rostral and caudal floor-plate, explants were cultured together with post-crossing commissural axons. One open-book preparation from a HH25 lumbosacral spinal cord was dissected into three explants containing commissural neurons and two floor-plate pieces (one rostral and one caudal to the commissural explants). The commissural neuron explants were randomly assigned to the three possible configurations, cultured for $20-24 \mathrm{~h}$, stained, and analyzed as described above.

\section{Results}

Several Wnts are expressed in the floor-plate area of the embryonic chicken spinal cord

Based on the identification of Wnt4 as a guidance cue for post-crossing commissural axons in the mouse spinal cord (Lyuksyutova et al., 2003), we analyzed its expression pattern in the chicken spinal cord during the time when axons of dorsolateral commissural neurons cross the floor plate and turn into the longitudinal axis. At the lumbosacral level of the spinal cord, axons have reached the floor-plate area at $\mathrm{HH} 22$ (Hamburger and Hamilton, 1951). At HH24, they turn into the longitudinal axis (Bourikas et al., 2005). During this time window, Wnt4 was expressed at high levels in the dorsal spinal cord but only at low levels in a small, narrow expression domain in the ventral spinal cord (supplemental Fig. S3, available at www.jneurosci.org as supplemental material). Unlike in the mouse, Wnt4 was not detectable in the chicken floor plate. Based on published expression patterns (Hollyday et al., 1995; Fokina and Frolova, 2006), Wnt1, Wnt2, Wnt3a, $W n t 6, W n t 9 a / b$, and Wnt16 could be excluded as well. Our own expression analysis by in situ hybridization excluded $W n t 5 b$, $W n t 7 b$, Wnt8a/c, Wnt9b, and Wnt11 (data not shown and supplemental Fig. S3, available at www.jneurosci.org as supplemental material).

$W n t 5 a$ and $W n t 7 a$ were expressed in a spatial and temporal pattern that was compatible with a role in post-crossing commissural axon guidance (supplemental Fig. S3, available at www. jneurosci.org as supplemental material). Wnt5a was found in the floor plate at both $\mathrm{HH} 22$ and $\mathrm{HH} 24$. Wnt7 $a$ was expressed adjacent to the floor plate in the area where post-crossing commissural axons turn into the longitudinal axis.

\section{Interference with Wnt5a and Wnt7a expression results in rostrocaudal pathfinding errors of post-crossing commissural axons}

To assess a possible function of Wnts in post-crossing commissural axon guidance, we used in ovo RNAi (Pekarik et al., 2003).
We injected dsRNA derived from Wnt4, Wnt5a, Wnt7a, or Wnt11 into the central canal of the spinal cord of embryonic day 3 chicken embryos (see supplemental Fig. S2, available at www. jneurosci.org as supplemental material, and Materials and Methods for details). Coinjection of a plasmid encoding EGFP was used to monitor the efficiency of nucleic acid transfer into the floor plate area (Bourikas et al., 2005). As a negative control, we used dsRNA derived from Wnt11, which was not expressed in the spinal cord (supplemental Fig. S3, available at www.jneurosci.org as supplemental material). Because antibodies for the targeted Wnts are not available, we assessed the specificity of downregulation by in situ hybridization (supplemental Fig. S1, available at www.jneurosci.org as supplemental material). In ovo RNAi resulted in a specific reduction of Wnt mRNA levels between 29 and $39 \%$ but did not change the patterning of the neural tube or interfere with commissural axons growth toward the floor plate (supplemental Fig. S4, available at www.jneurosci.org as supplemental material).

Upon downregulation of Wnt5a and Wnt7a, post-crossing axons failed to turn or made aberrant caudal turns as revealed by DiI tracing of dorsal commissural axons at the lumbosacral level of the spinal cord (Fig. 1). Many axons did not reach the contralateral floor-plate border in the absence of Wnt5a (Fig. 1C). This was not due to a delay of axon growth or a decreased growth rate, as axons in experimental and control embryos reached the floor plate at the same time. Furthermore, axons were still stuck in the floor plate when embryos lacking Wnt5a were analyzed at older stages (data not shown). 

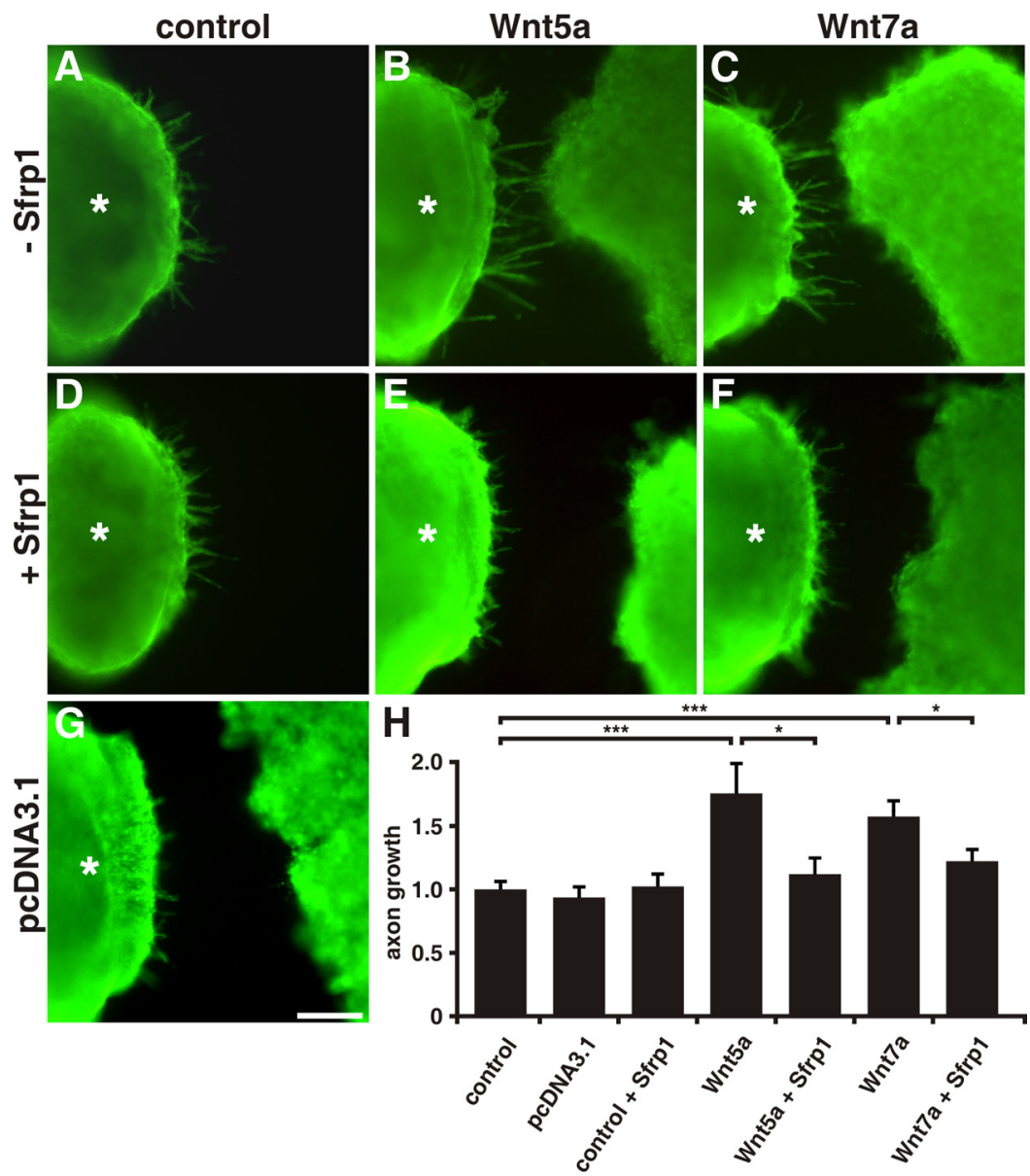

Figure 5. Sfrp1 blocks the attractive effect of Wnt5a and Wnt7a on post-crossing commissural axons. $\mathbf{A}-\mathbf{G}$, Explants of postcrossing commissural neurons (asterisk) were cultured alone or with COS cells expressing Wnt5a $(\boldsymbol{B}, \boldsymbol{E})$ or Wnt7a $(\boldsymbol{C}, \boldsymbol{F})$. COS cells transfected with the empty pcDNA3.1 vector had no effect on commissural axon growth $(0.94 \pm 0.08 \%, n=27)(\boldsymbol{G})$ (supplemental Table S3, available at www.jneurosci.org as supplemental material) compared with control explants cultured without $\mathrm{COS}$ cells $(1 \pm 0.06 \%, n=31)(\boldsymbol{A})$. In contrast, the presence of Wnt5a $(\boldsymbol{B})$ and Wnt7a $(\boldsymbol{C})$ considerably increased commissural axon growth $(1.76 \pm 0.24, n=21 ;$ and $1.57 \pm 0.13, n=23$, respectively). Adding $1 \mu \mathrm{g} / \mathrm{ml}$ Sfrp1 had no effect on post-crossing commissural explants $(1.02 \pm 0.10, n=19)(\boldsymbol{D})$. However, the presence of Sfrp1 significantly decreased the growth-promoting effect of Wnt5a $(1.12 \pm 0.13, n=20)(\boldsymbol{E})$ and Wnt7a $(1.22 \pm 0.1, n=24)(\boldsymbol{F})$, respectively. For statistical analysis the two-tailed Student's $t$ test was used $(\boldsymbol{H})$ (supplemental Table $\$ 3$, available at www.jneurosci.org as supplemental material). Values are given \pm SEM. ${ }^{* * *} p<0.001$ for Wnt5a and Wnt7a compared with control; ${ }^{*} p<0.05$ for Wnt5a and Wnt7a compared with Wnt5a + Sfrp1 and Wnt7a + Sfrp1, respectively. Scale bar, $200 \mu \mathrm{m}$.

To quantify the severity of the observed defects caused by downregulation of Wnt5a or Wnt7a, each injection site was classified into one of three phenotype categories (strong, weak, or none). A strong phenotype meant that $>50 \%$ of the axons stalled before reaching the contralateral floor-plate border or that fibers turned caudally along the longitudinal axis of the spinal cord. Caudal turns of dorsolateral commissural axons were never seen in control embryos. An injection site was scored as weak phenotype when $20-50 \%$ of the axons failed to cross the floor plate. Using these criteria, a strong phenotype was found at $34.9 \pm 7.2 \%$ of the injection sites in embryos lacking Wnt5a and at $27.9 \pm 8.1 \%$ of the injection sites in embryos lacking Wnt7a (Fig. 1 E; supplemental Table 1, available at www.jneurosci.org as supplemental material). In contrast, after downregulation of Wnt11 or Wnt4, the trajectories of post-crossing axons did not differ from noninjected or EGFP-expressing control embryos. If both weak and strong phenotypes were added, only $27.7 \%$ of the injection sites were normal in embryos lacking Wnt5a. After downregulation of
Wnt7a, 29.5\% of the injection sites were normal. In contrast, all control groups showed normal pathfinding behavior of commissural axons at $>70 \%$ of the injection sites.

Together, these results indicated that Wnts were involved in rostrocaudal pathfinding of post-crossing axons in the chicken spinal cord. However, in contrast to mouse, Wnt4 did not have an effect; instead, Wnt5a and Wnt7a were the Wnt family members necessary for post-crossing commissural axon guidance in the chicken embryo.

\section{Wnts are not expressed in a rostrocaudal gradient in the embryonic chicken spinal cord \\ Based on their function as guidance cues for post-crossing commissural axons in the chicken spinal cord and in analogy to obser- vations in the mouse (Lyuksyutova et al., 2003), we expected to find both Wnt5a and Wnt7a in a rostral ${ }^{\text {high }}$ to caudal ${ }^{\text {low }}$ gradient. Surprisingly, neither Wnt5a nor Wnt7a mRNA was found to be expressed in a gradient in the lumbosacral spinal cord (Fig. 2; supplemental Fig. S5, avail- able at www.jneurosci.org as supplemen- tal material). For the quantification, we used both transverse sections taken from different levels (data not shown) and open-book preparations of the lumbosa- cral spinal cord.}

\section{Sfrps are expressed in a rostrocaudal gradient in the embryonic chicken spinal cord}

To find an explanation for the apparent contradiction between functional data and Wht expression patterns, we turned to Sfrps. Addition of exogenous Sfrp was shown to antagonize Wnt activity on post-crossing commissural axons in cultures of mouse spinal cord explants (Lyuksyutova et al., 2003). Four Sfrp family members have been found in the chicken genome. In situ hybridization analysis indicated that three of the four Sfrps were expressed in the developing spinal cord between HH22 and HH26 (Fig. 3). Sfrp1 was expressed in the floor plate at high levels (Fig. $3 A, D)$ and most intriguingly exhibited a strong gradient along the longitudinal axis (Figs. 3G; supplemental Fig. S5, available at www.jneurosci.org as supplemental material). However, based on their expression patterns, a role for Sfrp 2 and Sfrp3 in postcrossing axon guidance would also be possible. Sfrp2 was expressed dorsal to the floor plate and in the ventral ventricular zone at both HH22 (Fig. 3B) and HH24 (Fig. 3E). Sfrp3 was expressed more widely in the spinal cord at HH22 (Fig. $3 C$ ) but decreased considerably thereafter. By HH24, expression was very low in the ventricular zone except for the area adjacent to the floor plate (Fig. $3 F$ ). Sfrp 4 was not expressed in the neural tube (data not shown). Sfrp2 (Figs. 3 H; supplemental Fig. S5, available at www.jneurosci.org as supplemental material) was expressed in a shallow gradient in contrast to Sfrp3 (Fig. 3I) that was expressed 
uniformly along the rostrocaudal axis. Based on their expression pattern, Sfrps made good candidates for regulators of Wnt activity in rostrocaudal guidance of post-crossing axons.

\section{Loss of Sfrp1 function results in rostrocaudal pathfinding errors of post-crossing axons}

To test for a role of Sfrps as antagonists of Wnt5a and Wnt7a in post-crossing axon guidance, we turned again to in ovo RNAi. Specific downregulation of Sfrp1 (supplemental Fig. S1, available at www.jneurosci. org as supplemental material) reproduced the loss-of-function phenotypes seen after silencing of Wnt5a and Wnt7a (Fig. 4). In the absence of Sfrp1, a strong phenotype, which is post-crossing axons turning caudally or $>50 \%$ of the axons stalling in the floor plate or at the floor-plate exit site, was found $27.5 \pm 5.2 \%$ of the DiI injection sites (Fig. 4; supplemental Table 2, available at www.jneurosci.org as supplemental material). Downregulation of Sfrp2 had a similar but weaker effect. Axons stalled or turned caudally at $18.3 \pm 6.7 \%$ of the injection sites. Downregulation of Sfrp3 and Sfrp4 did not affect commissural axon guidance, since strong phenotypes were observed at only $10.4 \pm 4.8 \%$ and $12.2 \pm 3.0 \%$ of the injection sites, respectively, compared with $11.1 \pm 5.0 \%$ in untreated controls. These results were consistent with our hypothesis that graded Wnt activity that attracted post-crossing axons rostrally was shaped by the graded expression of Sfrp1. However, based on these in vivo results, an additional direct role of Sfrps on postcrossing axons could not be excluded.

\section{Sfrp1 blocks the attractive effect of Wnt5a and Wnt7a on post-crossing commissural axons}

To distinguish between a direct and an indirect role of Sfrp1 on post-crossing axons, we turned to in vitro assays. Post-crossing commissural axons extended into the collagen matrix when spinal cord explants were cultured with the floor plate attached (Fig. $5)$. There was no difference in neurite growth when Sfrp1 was added (Fig. $5 D, H$ ). Post-crossing axons were significantly longer than controls when explants were cultured with COS cells expressing Wnt5a (Fig. 5B, H) or Wnt7a (Fig. 5C,H). The growthpromoting effect of both Wnt5a and Wnt7a was blocked in the presence of Sfrp 1 in the medium (Fig. $5 E, F$ ). Pre-crossing commissural axons extending from spinal cord explants did not respond to Wnt5a or Wnt7a (data not shown).

Our in vitro results excluded a direct effect of Sfrp1 on postcrossing axons and confirmed our in vivo observations, which suggested that an attractive effect of higher Wnt levels in the rostral spinal cord was generated by a graded expression of the Wnt antagonist Sfrp1. Additional evidence supporting this hypothesis was found in another in vitro assay where post-crossing commissural axons were exposed to rostral or caudal floor-plate explants (Fig. 6). Rostral floor-plate explants were more potent than caudal floor plate in promoting growth of post-crossing axons (Fig. $6 B-D$ ). As expected based on our previous assays, this effect could be blocked by Sfrp1 that was added to the culture medium (Fig. 6E-G).

Together, these experiments strongly supported our hypothesis that a graded activity of Wnts was achieved along the rostrocaudal axis of the embryonic chicken spinal cord by a graded expression of the Wnt antagonist Sfrp1.

\section{Overexpression of Wnts and Sfrps reverses the functional} Wnt gradients and causes aberrant behaviors of post-crossing axons

To provide further experimental evidence for our hypothesis in vivo, we performed gain-of-function experiments. We selectively overexpressed either Wnt5a or Wnt7a in the caudal spinal cord (Figs. 7; supplemental Fig. S6, available at www.jneurosci.org as supplemental material). Spatially controlled ectopic expression of Wnts at caudal levels was expected to exceed the capacity of the endogenous Sfrps to block Wnt function caudally (Fig. 7A). Conversely, overexpression of Sfrps at rostral levels was expected to disrupt the functional Wnt gradient by excessive levels of the antagonist (Fig. 7B). In agreement with the prediction (Fig. 7A), post-crossing axons randomly turned in either rostral or caudal 


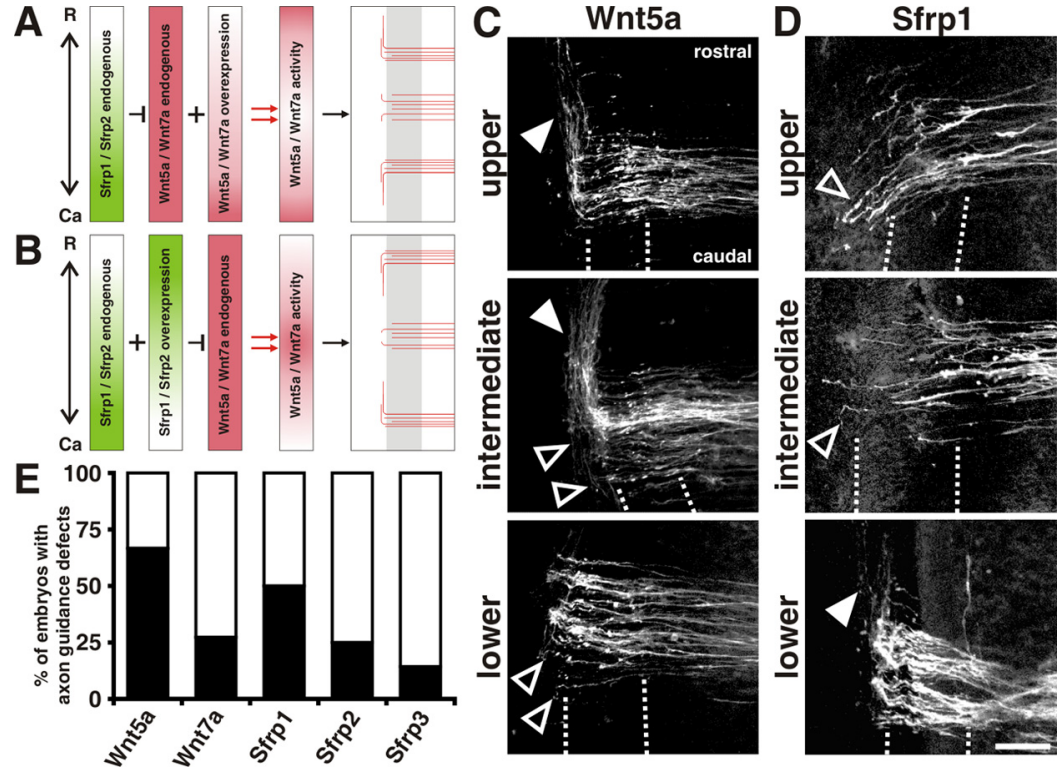

Figure 7. Reversal of the functional Wnt gradients by overexpression of either Wnts or Sfrps resulted in the expected anteroposterior guidance phenotypes. To test our hypothesis that a caudal ${ }^{\text {high }}$ to rostral ${ }^{\text {low }}$ Sfrp gradient was producing a Wnt activity gradient despite the homogenous rostrocaudal expression of Wnts, we used gain-of-function approaches. We reasoned that ectopic expression of Wnt5a or Wnt7a in the caudal lumbar spinal cord would result in a double-headed gradient with high Wnt activity levels in the thoracic (due to endogenous Wnt) and in the caudal spinal cord (due to overexpression). $\boldsymbol{A}$, The expected behavior of post-crossing axons is indicated schematically. $\boldsymbol{B}$, Alternatively, we predicted that ectopic expression of Sfrp1 or Sfrp2 in the thoracic spinal cord would block Wnt activity at thoracic and upper lumbosacral levels, and would therefore disrupt the Wnt activity gradient. In this case, post-crossing axons were expected to turn correctly in the caudal but not in the more rostral lumbosacral and thoracic spinal cord. $\boldsymbol{A}, \boldsymbol{C}, \boldsymbol{E}$, In our gain-of-function experiments, we observed the expected turning patterns $(\boldsymbol{A})$ in $67 \%$ of the embryos after ectopic expression of Wnt5a in the caudal spinal cord $(\boldsymbol{C}, \boldsymbol{E})$ [supplemental Fig. S6 (available at www.jneurosci.org as supplemental material) shows an overlay of the Dil-labeled axons with EGFP expression as a means to assess ectopic Wnt or Sfrp expression]. Overexpression of Wnt7a had a weaker effect (data not shown), resulting in the expected turning pattern in $27 \%$ of the embryos. $\boldsymbol{D}, \boldsymbol{B}, \boldsymbol{E}$, Alternatively, ectopic expression of Sfrp1 in the thoracic spinal cord (D) resulted in the expected turning pattern $(\boldsymbol{B})$ in $50 \%$ of the embryos $(\boldsymbol{E})$. $\boldsymbol{E}$, Overexpression of Sfrp2 (data not shown) had a weak effect, resulting in a change in the turning pattern in $25 \%$ of the embryos. Very little effect was found after ectopic expression of $S f r p 3$ ( $14 \%$ of the embryos exhibited changes). Rostral is to the top in $\boldsymbol{A}-\boldsymbol{D}$. The floor plate is indicated by dashed lines. Filled arrowheads indicate axons turning correctly in rostral direction. Open arrowheads indicate axons turning caudally. Scale bar, $60 \mu \mathrm{m}$.

direction or stalled at the exit site of the floor plate when Wnt5a was selectively overexpressed at caudal levels (Fig. 7C). When Sfrp1 was overexpressed at thoracic and upper lumbosacral levels of the spinal cord, post-crossing axons turned caudally at upper lumbosacral levels and mostly stalled at the floor-plate exit site at intermediate levels, whereas no change in the behavior was observed at caudal lumbosacral levels (Fig. $7 D$ ), again in agreement with the prediction (Fig. $7 B$ ). The same phenotypes were observed after caudal overexpression of $W n t 7 a$ and rostral expression of Sfrp2, although the effects were weaker than those observed after ectopic expression of $W n t 5 a$ and Sfrp1. The changes in axon guidance were not due to aberrant spinal cord patterning, as overexpression of Wnts or Sfrps at HH19 did not alter spinal cord patterning (supplemental Fig. S7, available at www.jneurosci.org as supplemental material).

For our gain-of-function analysis, we had to use a different quantification method compared with the loss-of-function experiments. Rather than plotting the average number of injection sites per embryo that showed a strong phenotype, we counted the embryos that exhibited the expected axon guidance pattern (Fig. $7 A, B)$ all along the lumbar spinal cord (Fig. $7 E$ ).

Caudal overexpression of $W n t 5 a$ resulted in the expected axon growth pattern (Fig. $7 A, C$ ) in $67 \%$ of the embryos (8 of $12 \mathrm{em}$ bryos). The effect of $W n t 7 a$ overexpression was much weaker and resulted in the expected phenotype in only three of 11 embryos.
Similarly, overexpression of Sfrp2 was less effective than Sfrp1 overexpression, with the expected pattern observed in 25 (3 of 12 ) and $50 \%$ (5 of 10$)$ of the embryos, respectively (Fig. $7 B, D$ ). Axonal navigation after Sfrp3 overexpression was affected in only one of seven embryos. The repulsive activity derived from the graded expression of Shh in the caudal spinal cord explains the failure to turn compared with the complete reversal of the growth direction that was observed for most axons (Bourikas et al., 2005).

In summary, our gain-of-function experiments support a model that predicts a Wnt activity gradient that is shaped by the graded expression of the Wnt antagonists, the Sfrps, as a guidance mechanism for post-crossing commissural axons.

\section{Shh indirectly regulates Wnt activity by inducing Wnt antagonists}

Because Shh induces Sfrp expression in mesenchymal tissue (Lee et al., 2000) and because Shh is expressed in the same caudal ${ }^{\text {high }}$ to rostral ${ }^{\text {low }}$ gradient along the longitudinal axis of the spinal cord as Sfrp1 (Fig. 3) (Bourikas et al., 2005), we tested whether Shh could also control Sfrp expression in the neural tube. Indeed, we found that ectopic expression of Shh in the developing spinal cord induced ectopic expression of Sfrp1 and Sfrp2, but not Sfrp3 (Fig. 8).

Together, our results suggest a model for post-crossing commissural axon guidance in the chicken spinal cord that is based on both Shh and Wnts. In contrast to the mouse, where Wnt4 was found to be expressed in a gradient in the floor plate, with high levels rostrally and low levels caudally, Wnt4 is not involved in post-crossing axon guidance in the chick. Rather, Wnt5a and Wnt7a direct axons rostrally upon floor plate exit. However, neither $W n t 5 a$ nor $W n t 7 a$ were found to be expressed in a gradient comparable to the one found for Wnt4 in the mouse. Thus, in the chicken spinal cord, a functional Wnt gradient, rather than an expression gradient, attracts post-crossing commissural axons rostrally. The graded activity is achieved by a graded expression of the Wnt antagonist Sfrp1 and, to a lesser extent, Sfrp2. The expression of Sfrpl appears to be regulated by the graded expression of Shh that was shown to have a repulsive effect on postcommissural axons on its own (Bourikas et al., 2005). These results suggest that axons are pushed rostrally by the repellent activity of Shh and attracted rostrally by the graded Wnt activity that is achieved by increasing blockade of Wnt activity in the caudal spinal cord (Fig. 8). Thus, Shh appears to act as a guidance cue for post-crossing commissural axons directly by repelling them and indirectly by inducing the expression of Sfrps that shape a gradient of attractive Wnt activity.

\section{Discussion}

In a previous study, we identified a role of the morphogen Shh in post-crossing commissural axon guidance in the chicken spinal cord (Bourikas et al., 2005). In contrast to pre-crossing commis- 


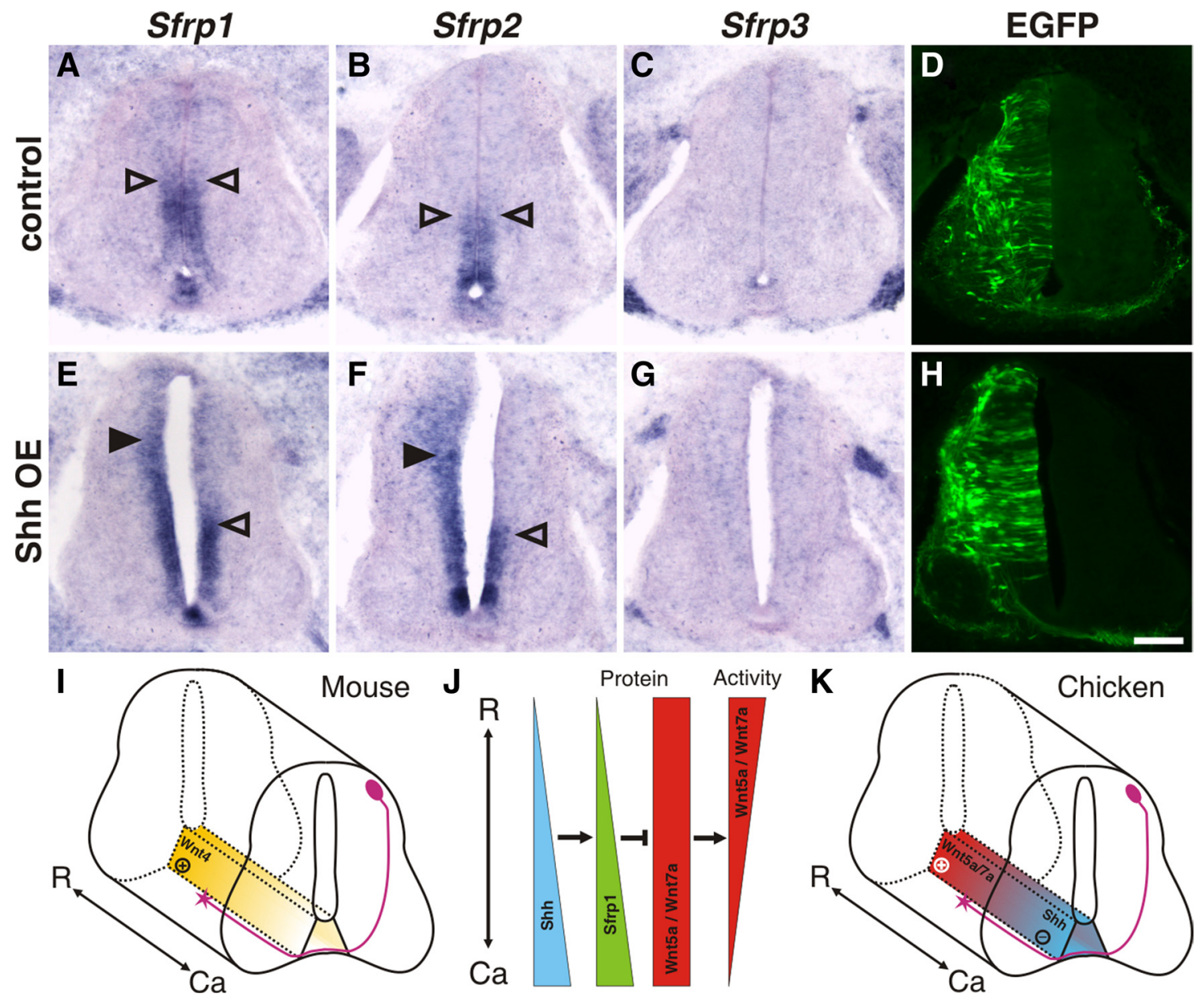

Figure 8. Shh induces expression of Sfrp1 and Sfrp2. $\boldsymbol{A}-\boldsymbol{H}$, The expression patterns of $\operatorname{Sfrp} 1(\boldsymbol{A}, \boldsymbol{E}), \operatorname{Sfrp2}(\boldsymbol{B}, \boldsymbol{F})$, and $\operatorname{Sfrp} 3(\boldsymbol{C}, \boldsymbol{G})$ are shown in transverse sections of embryos injected with the EGFP plasmid only $(\boldsymbol{A}-\boldsymbol{D})$ and embryos coinjected with expression plasmids for Shh and $E G F P(\boldsymbol{E}-\boldsymbol{H})$. Overexpression (OE) of Shh resulted in the induction of $\operatorname{Sfrp} 1$ ( $\boldsymbol{E}$, arrowhead) and $\operatorname{Sfrp2}(\boldsymbol{F}$, arrowhead) on the electroporated (left) side compared with the nonelectroporated side. Open arrowheads on the left side indicate the absence of Sfrp expression changes in the corresponding area of a control-injected embryo expressing only EFGP but no ectopic Shh. No induction of Sfrp3 was found after overexpression of Shh (G) compared with control-injected embryos (C). Scale bar, $100 \mu \mathrm{m}$. $\boldsymbol{I}-\boldsymbol{K}$, Wnts control post-crossing commissural axon guidance by different mechanisms in mouse and chicken spinal cords. $\boldsymbol{I}, \mathrm{A}$ transcriptional gradient of Wnt 4 in the floor plate was found to attract post-crossing commissural axons rostrally in the mouse spinal cord (Lyuksyutova et al., 2003). J, In the chicken embryo, Wnt5a and Wnt7a are not expressed in a gradient along the anteroposterior axis of the spinal cord. Rather, a Wnt activity gradient is shaped by the graded expression of Sfrp1 that in turn is induced by the graded expression of Shh (Bourikas et al., 2005). $\boldsymbol{K}$, The resulting gradient of Wnt function with high activity rostrally and low activity caudally cooperates with the repellent activity of Shh that is expressed in a rostral ${ }^{\text {low }}$ to caudal ${ }^{\text {high }}$ gradient to direct post-crossing commissural axons along the longitudinal axis of the spinal cord. R, Rostral; Ca, caudal.

sural axon guidance, where Shh was shown to act as a chemoattractant in parallel to Netrin-1 (Charron et al., 2003; Yam et al., 2009), the activity of Shh was not mediated by Smoothened and Boc but rather by Hhip. Consistent with its expression pattern, Shh acts as a repellent for post-crossing axons (Bourikas et al., 2005). In contrast, an attractive effect on post-crossing axons was demonstrated for Wnt4 in the mouse (Lyuksyutova et al., 2003), raising the question whether the two species use different morphogens for this navigational task or whether Shh and Wnts would cooperate (Stoeckli, 2006). In this study, we show that the role of Wnts is conserved in the chicken spinal cord but with important mechanistic differences. In the chick, Wnt5a and Wnt7a, rather than Wnt4, direct post-crossing axons rostrally upon floor-plate exit. But most importantly, in contrast to the mouse, where Wnt4 was expressed in a gradient with high levels in the rostral and low levels in the caudal floor plate, expression levels of $W n t 5 a$ and $W n t 7 a$ did not change along the anteroposterior axis of the chicken spinal cord (supplemental Fig. S5, available at www.jneurosci.org as supplemental material). Still, loss of Wnt5a or Wnt7a function resulted in aberrant navigation of post-crossing axons along the contralateral floor-plate border (Fig. 1). This apparently contradictory finding was explained by a graded expression of Sfrp1 that established a functional Wnt gradient with high levels rostrally and low levels caudally (supplemental Fig. S5, available at www.jneurosci.org as supplemental material). Sfrps are known antagonists of Wnts (Jones and Jomary, 2002; Kawano and Kypta, 2003). Moreover, Sfrps were shown to interfere with the role of Wnt4 in post-crossing axon guidance when added to cultures of mouse spinal cord explants (Lyuksyutova et al., 2003). However, it is not known whether Sfrps are required for post-crossing commissural axon guidance in the mouse in vivo, or if the transcriptional gradient of Wnt4 is sufficient.

Navigation of retinal ganglion cell axons was demonstrated to be directly influenced by Sfrp1 in both chick and frog (Rodriguez et al., 2005). However, we found no evidence for a direct effect of Sfrp1 on post-crossing commissural axons (Fig. 5). Rather, Sfrp1 blocked the attractive effect of Wnt5a and Wnt7a on postcrossing commissural axons both in vitro (Figs. 5 and 6) and in vivo (Fig. 7). Selective overexpression of Sfrp1 rostrally, i.e., at 
thoracic and upper lumbosacral levels, interfered with the rostral turn of post-crossing axons. The effect was pronounced at rostral levels of the lumbosacral spinal cord. There, the relative contribution of Wnts to post-crossing axon guidance is expected to be stronger than more caudally, where axons are also repelled by Shh. Consistent with the expected Wnt activity levels along the anteroposterior axis (Fig. 7), caudal turns of post-crossing axons were found in rostral segments but not intermediate or caudal segments of the lumbosacral spinal cord, where axons were mostly stalled at the floor-plate exit site. Similarly, rostrocaudal axon pathfinding was perturbed after overexpression of Wnt5a in the caudal floor plate. We do not know why overexpression of Wnt7a was less effective in our gain-of-function assay, as downregulation of Wnt5a and Wnt7a induced rostrocaudal pathfinding errors to a similar extent. A possible explanation would be that overexpression levels of Wnt7a were lower than those reached after ectopic expression of Wnt5a.

Together, our observations made in both loss- and gain-offunction experiments support a model where a functional Wnt gradient is shaped by a graded inhibition of Wnts rather than by the control of their transcription.

At present, it is unclear how Wnt signaling mediates postcrossing commissural axon guidance. Studies in the chicken hindbrain have linked Wnt5a and PCP signaling to motoneuron migration (Vivancos et al., 2009). Studies in mouse have ruled out the canonical pathway, because anteroposterior guidance was normal in Lrp6 knock-out mice (Lyuksyutova et al., 2003). Instead, the effect of Wnt4 on post-crossing axons required atypical protein kinase C (Wolf et al., 2008). Transcription-independent signaling was consistent with findings for Shh, where transcription is not required for its chemoattractive effect, despite the fact that signaling is still mediated by Patched and Smoothened (Yam et al., 2009). In agreement with previous findings for Shh (Bourikas et al., 2005), we did not observe an effect on neural tube patterning when either Wnt5a or Wnt7a were downregulated or overexpressed during the time window of commissural axon pathfinding, indicating that Wnt affected axon guidance directly (supplemental Figs. S4, S7, available at www.jneurosci.org as supplemental material).

In mouse, Wnt5a was shown to have a repulsive effect on corticospinal axons (Liu et al., 2005) and on cortical axons forming the corpus callosum (Keeble et al., 2006). This effect required Ryk, an atypical receptor tyrosine kinase. Recently, Wnt5a was shown to affect axon growth through activation of Ryk, whereas turning away from a Wnt5a source required both Ryk and Frizzled2 signaling (Li et al., 2009). Signaling downstream of Ryk is not well characterized (Endo and Rubin, 2007). Signaling activated by Wnt5a is generally thought to be noncanonical (Qian et al., 2007), but activation of the $\beta$-catenin signaling pathway in a context-dependent manner has also been found (Mikels and Nusse, 2006). In contrast, Wnt7a is generally associated with canonical Wnt signaling. Therefore, additional experiments will be required to sort out downstream signaling components of Wnts in axon guidance.

Interestingly, the two guidance cues for post-crossing axons in chicken, Shh and Wnts, do not act independently. As shown previously, Shh directly repels post-crossing axons in vitro and thus, consistent with its graded expression in the floor plate, pushes post-crossing commissural axons rostrally (Bourikas et al., 2005). In addition, Shh affects Wnt activity by indirectly shaping the functional gradient of Wnts via the induction of the Wnt antagonists Sfrp1 and Sfrp2. We tested for such a scenario due to reports in the literature, where Shh-induced upregulation of Sfrp2 was found to compete with Wnt1 and Wnt4 in establishing dorsoventral polarity of the somitic mesoderm (Lee et al., 2000). Because, in the chicken floor-plate area, Sfrp1 was expressed in a gradient that was similar to the Shh expression gradient (Fig. 3, supplemental Fig. S5, available at www.jneurosci.org as supplemental material), we analyzed whether Shh could regulate the expression of Sfrps and thus control the functional gradient of Wnt5a and Wnt7a (Fig. 8). Indeed, we could demonstrate that overexpression of Shh induced ectopic expression of Sfrp 1 and Sfrp2, but not Sfrp3 (Fig. 8), in agreement with an indirect effect of Shh on Wnt activity via induction of Sfrp1 expression.

The opposite experiment, blocking Sfrp expression by downregulation of Shh, was not possible because of time constraints. Downregulation of Shh in 3-d-old embryos does not block expression of Sfrps, consistent with the strong expression of Sfrp1 in the floor plate already at HH18. However, downregulation of Shh at early stages of spinal cord development does not allow for the assessment of Sfrp expression, due to the well known morphogen activity of Shh that is required for the patterning of the neural tube.

At present, the mechanism by which Shh regulates Sfrp expression is not known. Ectopic expression of Shh induced expression of both Sfrp1 and Sfrp2. Because endogenous Sfrp2 is not expressed in the floor plate but rather in cells adjacent to it, a paracrine mechanism of induction by Shh has to be postulated. However, an additional autocrine mechanism cannot be excluded for the induction of Sfrp1, which is expressed in the floor plate and, thus, in the same cells that express Shh. Our experiments did not allow us to distinguish between the two mechanisms. Additional studies will be required to address this issue and also to test whether signaling components in commissural neurons are shared between Shh and Wnts. Despite the fact that Shh has a major effect on Wnt activity by shaping a Wnt activity gradient, additional crosstalk at the level of surface receptors or intracellular signaling components cannot be excluded.

In summary, our results support a model for postcommissural axon guidance in the chicken embryo that implicates Shh directly as a repellent and indirectly as a regulator of Wnt activity by its effect on the graded expression of the Wnt antagonist Sfrp1.

\section{References}

Avraham O, Hadas Y, Vald L, Zisman S, Schejter A, Visel A, Klar A (2009) Transcriptional control of axonal guidance and sorting in dorsal interneurons by the Lim-HD proteins Lhx9 and Lhx1. Neural Dev 4:21.

Bourikas D, Pekarik V, Baeriswyl T, Grunditz A, Sadhu R, Nardó M, Stoeckli ET (2005) Sonic hedgehog guides commissural axons along the longitudinal axis of the spinal cord. Nat Neurosci 8:297-304.

Charron F, Stein E, Jeong J, McMahon AP, Tessier-Lavigne M (2003) The morphogen sonic hedgehog is an axonal chemoattractant that collaborates with netrin-1 in midline axon guidance. Cell 113:11-23.

Endo Y, Rubin JS (2007) Wnt signaling and neurite outgrowth: insights and questions. Cancer Sci 98:1311-1317.

Esteve P, Trousse F, Rodríguez J, Bovolenta P (2003) SFRP1 modulates retina cell differentiation through a beta-catenin-independent mechanism. J Cell Sci 116:2471-2481.

Fokina VM, Frolova EI (2006) Expression patterns of Wnt genes during development of an anterior part of the chicken eye. Dev Dyn 235:496-505.

Hamburger V, Hamilton HL (1951) A series of normal stages in the development of the chick embryo. J Morphol 88:49-92.

Hollyday M, McMahon JA, McMahon AP (1995) Wnt expression patterns in chick embryo nervous system. Mech Dev 52:9-25.

Jones SE, Jomary C (2002) Secreted Frizzled-related proteins: searching for relationships and patterns. Bioessays 24:811-820.

Kawano Y, Kypta R (2003) Secreted antagonists of the Wnt signalling pathway. J Cell Sci 116:2627-2634.

Keeble TR, Halford MM, Seaman C, Kee N, Macheda M, Anderson RB, 
Stacker SA, Cooper HM (2006) The Wnt receptor Ryk is required for Wnt5a-mediated axon guidance on the contralateral side of the corpus callosum. J Neurosci 26:5840-5848.

Lee CS, Buttitta LA, May NR, Kispert A, Fan CM (2000) SHH-N upregulates Sfrp2 to mediate its competitive interaction with WNT1 and WNT4 in the somitic mesoderm. Development 127:109-118.

Li L, Hutchins BI, Kalil K (2009) Wnt5a induces simultaneous cortical axon outgrowth and repulsive axon guidance through distinct signaling mechanisms. J Neurosci 29:5873-5883.

Liu Y, Shi J, Lu CC, Wang ZB, Lyuksyutova AI, Song X, Zou Y (2005) Rykmediated Wnt repulsion regulates posterior-directed growth of corticospinal tract. Nat Neurosci 8:1151-1159.

Lyuksyutova AI, Lu CC, Milanesio N, King LA, Guo N, Wang Y, Nathans J, Tessier-Lavigne M, Zou Y (2003) Anterior-posterior guidance of commissural axons by Wnt-frizzled signaling. Science 302:1984-1988.

Mauti O, Sadhu R, Gemayel J, Gesemann M, Stoeckli ET (2006) Expression patterns of plexins and neuropilins are consistent with cooperative and separate functions during neural development. BMC Dev Biol $6: 32$.

Mauti O, Domanitskaya E, Andermatt I, Sadhu R, Stoeckli ET (2007) Semaphorin6A acts as a gate keeper between the central and the peripheral nervous system. Neural Dev 2:28.

Mikels AJ, Nusse R (2006) Purified Wnt5a protein activates or inhibits betacatenin-TCF signaling depending on receptor context. PLoS Biol 4:e115.

Niederkofler V, Baeriswyl T, Ott R, Stoeckli ET (2010) Nectin-like molecules/SynCAMs are required for post-crossing commissural axon guidance. Development 137:427-435.

Okada A, Charron F, Morin S, Shin DS, Wong K, Fabre PJ, Tessier-Lavigne M, McConnell SK (2006) Boc is a receptor for sonic hedgehog in the guidance of commissural axons. Nature 444:369-373.
Pekarik V, Bourikas D, Miglino N, Joset P, Preiswerk S, Stoeckli ET (2003) Screening for gene function in chicken embryo using RNAi and electroporation. Nat Biotechnol 21:93-96.

Perrin FE, Stoeckli ET (2000) Use of lipophilic dyes in studies of axonal pathfinding in vivo. Microsc Res Tech 48:25-31.

Perrin FE, Rathjen FG, Stoeckli ET (2001) Distinct subpopulations of sensory afferents require F11 or axonin-1 for growth to their target layers within the spinal cord of the chick. Neuron 30:707-723.

Qian D, Jones C, Rzadzinska A, Mark S, Zhang X, Steel KP, Dai X, Chen P (2007) Wnt5a functions in planar cell polarity regulation in mice. Dev Biol 306:121-133.

Rodriguez J, Esteve P, Weinl C, Ruiz JM, Fermin Y, Trousse F, Dwivedy A, Holt C, Bovolenta P (2005) SFRP1 regulates the growth of retinal ganglion cell axons through the Fz2 receptor. Nat Neurosci 8:1301-1309.

Stoeckli ET (2006) Longitudinal axon guidance. Curr Opin Neurobiol $16: 35-39$.

Stoeckli ET, Landmesser LT (1995) Axonin-1, Nr-CAM, and Ng-CAM play different roles in the in vivo guidance of chick commissural neurons. Neuron 14:1165-1179.

Vivancos V, Chen P, Spassky N, Qian D, Dabdoub A, Kelley M, Studer M, Guthrie S (2009) Wnt activity guides facial branchiomotor neuron migration, and involves the PCP pathway and JNK and ROCK kinases. Neural Dev 4:7.

Wolf AM, Lyuksyutova AI, Fenstermaker AG, Shafer B, Lo CG, Zou Y (2008) Phosphatidylinositol-3-kinase-atypical protein kinase $\mathrm{C}$ signaling is required for Wnt attraction and anterior-posterior axon guidance. J Neurosci 28:3456-3467.

Yam PT, Langlois SD, Morin S, Charron F (2009) Sonic hedgehog guides axons through a noncanonical, Src-family-kinase-dependent signaling pathway. Neuron 62:349-362. 